\title{
Numerical Simulation of Acoustic-Gravity Waves Propagation in a Heterogeneous Earth-Atmosphere Model with Wind in the Atmosphere
}

\author{
B. G. Mikhailenko, A. A. Mikhailov, G. V. Reshetova \\ The Institute of Computational Mathematics and Mathematical Geophysics, \\ Siberian Branch of the RAS, pr. Akad. Lavrentieva, 6, Novosibirsk 630090, Russia \\ Email:mikh@sscc.ru \\ Received July 2013
}

\begin{abstract}
A numerical-analytical solution for seismic and acoustic-gravity waves propagation is applied to a heterogeneous "Earth-Atmosphere" model. Seismic wave propagation in an elastic half-space is described by a system of first order dynamic equations of elasticity theory. Propagation of acoustic-gravity waves in the atmosphere is described by the linearized Navier-Stokes equations with the wind. The algorithm proposed is based on the integral Laguerre transform with respect to time, the finite integral Fourier transform along the spatial coordinate with the finite difference solution of the reduced problem.
\end{abstract}

Keywords: Seismic Waves; Acoustic-Gravity Waves; Navier-Stokes Equations; Laguerre Transform; Finite Difference Method

\section{Introduction}

In mathematical simulation of seismic wave fields in an elastic medium, it is typically assumed that the medium borders on vacuum, and boundary conditions are specified on a free surface. Specifically, at the boundary, seismic waves are assumed to be absolutely reflected, and the generation of acoustic-gravity waves by elastic waves in the atmosphere and their interaction at the boundary are ignored.

In the last decade, some theoretical and experimental investigations have shown that there is a striking correlation between the waves in the lithosphere and the atmosphere. Paper [1] describes the effect of acoustoseismic induction of an acoustic wave produced by a vibrator. Papers [2,3] deal with theoretical investigations of wave processes at the boundary between an elastic half-space and an isothermal homogeneous atmosphere. In these papers, properties of the surface Stoneley-Scholte and modified Lamb waves are studied.

In the present paper we consider an efficient numerical algorithm to simulate and investigate the propagation of seismic and acoustic-gravity waves in a spatially inhomogeneous "Atmosphere-Earth" model. A peculiarity of the algorithm is a combination of integral transforms with a finite-difference method.

A similar approach to solving the problem for a verti- cally inhomogeneous model in a cylindrical system of coordinates with no wind in the atmosphere was considered in [4]. In the problem statement, the initial system is written down as a first-order hyperbolic system in terms of the velocity vector and stress tensor in a 3D Cartesin system of coordinates. The medium parameters are assumed to be functions of only two coordinates, and the medium is assumed to be homogeneous in the third coordinate. This problem statement is called a $2.5 \mathrm{D}$ one. The algorithm is based on the integral Laguerre transform with respect to the temporal coordinate. This method can be considered to be an analog to a well-known spectral method based on the Fourier transform, where, instead of the frequency $\omega$, we have a parameter $p$ that is the degree of the Laguerre polynomials. The integral Laguerre transform with respect to time (in contrast to the Fourier transform) makes it possible to reduce the initial problem to solving a system of equations in which the parameter is present only in the right-hand side of the equations and has a recurrence relation. This method for solving dynamic problems of elasticity theory was first considered in papers $[5,6]$ and then developed for problems of viscoelasticity $[7,8]$ and porous media [9]. The above-mentioned papers consider peculiarities of this method and the advantages of the integral Laguerre transform over the difference methods and the Fourier transform with respect to time. 


\section{Problem Statement}

The system of equations for the propagation of acoustic-gravity waves in an inhomogeneous non-ionized isothermal atmosphere in the Cartesian system of coordinates $(x, y, z)$ with the wind directed along the horizontal axis $x$ and vertical stratification along the axis $z$ has the following form:

$$
\begin{gathered}
\frac{\partial u_{x}}{\partial t}+v_{x} \frac{\partial u_{x}}{\partial x}=-\frac{1}{\rho_{0}} \frac{\partial P}{\partial x}-u_{z} \frac{\partial v_{x}}{\partial z}, \\
\frac{\partial u_{y}}{\partial t}+v_{x} \frac{\partial u_{y}}{\partial x}=-\frac{1}{\rho_{0}} \frac{\partial P}{\partial y}, \\
\frac{\partial u_{z}}{\partial t}+v_{x} \frac{\partial u_{z}}{\partial x}=-\frac{1}{\rho_{0}} \frac{\partial P}{\partial z}-\frac{\rho g}{\rho_{0}}, \\
\frac{\partial P}{\partial t}+v_{x} \frac{\partial P}{\partial x}=c_{0}^{2}\left[\frac{\partial \rho}{\partial t}+v_{x} \frac{\partial \rho}{\partial x}+u_{z} \frac{\partial \rho_{0}}{\partial z}\right]-u_{z} \frac{\partial P_{0}}{\partial z} \\
\frac{\partial \rho}{\partial t}+v_{x} \frac{\partial \rho}{\partial x}=-\rho_{0}\left[\frac{\partial u_{x}}{\partial x}+\frac{\partial u_{y}}{\partial y}+\frac{\partial u_{z}}{\partial z}\right] \\
-u_{z} \frac{\partial \rho_{0}}{\partial z}+F(x, y, z, t)
\end{gathered}
$$

Here $g$ is the acceleration of gravity, $\rho_{0}(z)$ is the reference atmosphere density, $c_{0}(z)$ is the sound speed, $v_{x}(z)$ is the wind velocity along the axis $x$, $\boldsymbol{u}=\left(u_{x}, u_{y}, u_{z}\right)$ is the velocity vector of displacement of the air particles, $P$ and $\rho$ are the pressure and the density perturbations, respectively, due to a wave propagating from a source of mass $F(x, y, z, t)=\delta\left(r-r_{0}\right) f(t)$, where $f(t)$ is a given time signal in the source. Assume that the axis $z$ is directed upwards. Zero subscripts for the medium physical parameters show their values for the reference atmosphere. The atmospheric pressure $P_{0}$ and the density $\rho_{0}$ for the reference atmosphere in a homogeneous gravitational field are:

$$
\frac{\partial P_{0}}{\partial z}=-\rho_{0} g, \quad \rho_{0}(z)=\rho_{1} \exp (-z / H),
$$

where $H$ is the height of the isothermal homogeneous atmosphere, and $\rho_{1}$ is the density of the atmosphere at the Earth's surface, that is, at $z=0$.

The seismic waves propagation in an elastic medium is described by the well-known system of first order equations of elasticity theory as the following relation between the displacement velocity vector components and the stress vector components:

$$
\begin{array}{r}
\frac{\partial u_{i}}{\partial t}=\frac{1}{\rho_{0}} \frac{\partial \sigma_{i k}}{\partial x_{k}}+F_{i} f(t), \\
\frac{\partial \sigma_{i k}}{\partial t}=\mu\left(\frac{\partial u_{k}}{\partial x_{i}}+\frac{\partial u_{i}}{\partial x_{k}}\right)+\lambda \delta_{i k} d i v \boldsymbol{u} .
\end{array}
$$

Here $\delta_{i j}$ is the Kronecker symbol, $\lambda\left(x_{1}, x_{2}, x_{3}\right)$ and $\mu\left(x_{1}, x_{2}, x_{3}\right)$ are the elastic parameters of the medium, $\rho_{0}\left(x_{1}, x_{2}, x_{3}\right)$ is the density, $\boldsymbol{u}=\left(u_{1}, u_{2}, u_{3}\right)$ is the displacement velocity vector, and $\sigma_{i j}$ are the stress vector components. The equality $\boldsymbol{F}(x, y, z)=F_{1} \boldsymbol{e}_{x}+F_{2} \boldsymbol{e}_{y}+F_{3} \boldsymbol{e}_{z}$ describes the distribution of a source located in space, and $f(t)$ is a given time signal in the source.

The combined system of equations for the propagation of seismic and acoustic-gravity waves in the Cartesian system of coordinates $(x, y, z)=\left(x_{1}, x_{2}, x_{3}\right)$ can be written down as

$$
\begin{gathered}
\frac{\partial u_{i}}{\partial t}=\frac{1}{\rho_{0}} \frac{\partial \sigma_{i k}}{\partial x_{k}}+F_{i} f(t)-K\left[v_{x} \frac{\partial u_{i}}{\partial x_{1}}+\frac{\rho g}{\rho_{0}} e_{z}-u_{z} \frac{\partial v_{x}}{\partial x_{3}} e_{x}\right], \\
\frac{\partial \sigma_{i k}}{\partial t}=\mu\left(\frac{\partial u_{k}}{\partial x_{i}}+\frac{\partial u_{i}}{\partial x_{k}}\right)+\lambda \delta_{i k} \operatorname{div} \boldsymbol{u}-\delta_{i k} K\left[v_{x} \frac{\partial \sigma_{i k}}{\partial x_{1}}+\rho_{0} g u_{z}\right] \\
K\left[\frac{\partial \rho}{\partial t}+v_{x} \frac{\partial \rho}{\partial x}=-\rho_{0} \operatorname{div} \boldsymbol{u}-u_{z} \frac{\partial \rho_{0}}{\partial z}\right] .
\end{gathered}
$$

Here $\delta_{i j}$ is the Kronecker symbol, $\rho_{0}(x, z)$ is the density, $\lambda(x, z)$ and $\mu(x, z)$ are the elastic parameters of the medium, $\vec{u}=\left(u_{1}, u_{2}, u_{3}\right)$ is the displacement velocity vector, and $\sigma_{i j}$ are the stress tensor components; $\boldsymbol{F}(x, y, z)=F_{1} \boldsymbol{e}_{x}+F_{2} \boldsymbol{e}_{y}+F_{3} \boldsymbol{e}_{z}$ describes the distribution of a source located in space, and $f(t)$ is a given time signal in the source. The medium is assumed to be homogeneous along the axis $y$.

System (1)-(5) for the atmosphere is obtained from system (8)-(10) at

$$
\begin{array}{r}
K_{\text {amм }}=1, \sigma_{12}=\sigma_{13}=\sigma_{23}=0, \sigma_{11}=\sigma_{22}=\sigma_{33}=-P, \\
\lambda=c_{0}^{2} \rho_{0}, \mu=0 .
\end{array}
$$

Set $K_{\text {amu }}=0$ in system (9)-(10), and obtain the system of Equations (6)-(7) for the propagation seismic waves in an elastic medium.

In the problem in question, the atmosphere-elastic half-space interface is assumed to be the plane $z=x_{3}=0$. In this case, the condition of contact of the two media at $z=0$ is written as

$$
\begin{array}{r}
\left.u_{z}\right|_{z=-0}=\left.u_{z}\right|_{z=+0},\left.\frac{\partial \sigma_{z z}}{\partial t}\right|_{z=-0}=\left.\left(\frac{\partial \sigma_{z z}}{\partial t}+\rho_{0} g u_{z}\right)\right|_{z=+0}, \\
\left.\sigma_{x z}\right|_{z=-0}=\left.\sigma_{y z}\right|_{z=-0}=0 .
\end{array}
$$

The problem is solved at the following zero initial data:

$$
\left.u_{i}\right|_{t=0}=\left.\sigma_{i j}\right|_{t=0}=\left.P\right|_{t=0}=\left.\rho\right|_{t=0}=0, i=1,2,3 . j=1,2,3 .
$$

All the functions of the wave field components are assumed to be sufficiently smooth so that the transforma- 
tions presented below be valid.

\section{Solution algorithm}

At the first step, we use the finite cosine-sine Fourier transform with respect to the spatial coordinate $y$, where the medium is assumed to be homogeneous. For each component of the system, we introduce the corresponding cosine or sine transform:

$$
\begin{array}{r}
W(x, z, n, t)=\int_{0}^{a} \boldsymbol{W}(x, y, z, t)\left\{\begin{array}{c}
\cos \left(k_{n} y\right) \\
\sin \left(k_{n} y\right)
\end{array}\right\} d(y), \\
n=0,1,2, \ldots, N ;(13)
\end{array}
$$

with the corresponding inversion formula

$$
\boldsymbol{W}(x, y, z, t)=\frac{1}{\pi} \boldsymbol{W}(x, 0, z, t)+\frac{2}{\pi} \sum_{n=1}^{N} \boldsymbol{W}(x, n, z, t) \cos \left(k_{n} y\right)
$$

or

$$
\boldsymbol{W}(x, y, z, t)=\frac{2}{\pi} \sum_{n=1}^{N} \boldsymbol{W}(x, n, z, t) \sin \left(k_{n} y\right)
$$

where $k_{n}=\frac{n \pi}{a}$.

At rather a large distance $a$, consider a wave field up to the time $t<T$, where $T$ is a minimum propagation time of a pressure wave to the boundary $r=a$. As a result of this transformation, we obtain $N+1$ independent 2D unsteady problems.

At the second step, we apply to the thus obtained $N+1$ independent problems the integral Laguerre transform with respect to time

$$
\begin{gathered}
\boldsymbol{W}_{p}(x, n, z)=\int_{0}^{\infty} \boldsymbol{W}(x, n, z, t)(h t)^{-\frac{\alpha}{2}} l_{p}^{\alpha}(h t) d(h t), \\
p=0,1,2, \ldots
\end{gathered}
$$

with the inversion formula

$$
\boldsymbol{W}(x, n, z, t)=(h t)^{\frac{\alpha}{2}} \sum_{p=0}^{\infty} \frac{p !}{(p+\alpha) !} \boldsymbol{W}_{p}(x, n, z) l_{p}^{\alpha}(h t),
$$

where $l_{p}^{\alpha}(h t)$ are the orthogonal Laguerre functions.

The Laguerre functions $l_{p}^{\alpha}(h t)$ can be expressed in terms of the classical standard Laguerre polynomials $L_{p}^{\alpha}(h t)$ (see paper [10]). Here we select an integer parameter $\alpha \geq 1$ to satisfy the initial data and introduce the shift parameter $h>0$. Then we have the following representation:

$$
l_{p}^{\alpha}(h t)=(h t)^{\alpha / 2} \exp (-h t / 2) L_{p}^{\alpha}(h t) .
$$

We take the finite cosine-sine Fourier transform with respect to the coordinate $x$, similar to the previous transform with respect to the coordinate $y$ with the corresponding inversion formulas:

$$
\begin{aligned}
\boldsymbol{W}_{p}\left(x, n, z_{i}\right)= & \frac{1}{\pi} \boldsymbol{W}_{p}\left(0, n, z_{i}\right) \\
& +\frac{2}{\pi} \sum_{m=1}^{M} \boldsymbol{W}_{p}\left(m, n, z_{i}\right) \cos \left(k_{m} x\right)
\end{aligned}
$$

or

$$
\boldsymbol{W}\left(x, n, z_{i}, p\right)=\frac{2}{\pi} \sum_{m=1}^{M} \boldsymbol{W}\left(m, n, z_{i}, p\right) \sin \left(k_{m} x\right),
$$

where $k_{m}=\frac{m \pi}{b}$.

It should be noted that the medium in this direction is inhomogeneous.

The finite difference approximation for the system of linear algebraic equations with respect to $z$ using the staggered grid method was applied (see paper [11]) providing second order accuracy approximation. This scheme is used for FD approximation within the computation domains in the atmosphere and in the elastic half-space, the fitting conditions at the interface being exactly satisfied. As a result of the above transformations, we obtain $N+1$ systems of linear algebraic equations, where $N$ is the number of harmonics in the Fourier transform with respect to the coordinate $y$.

The sought for solution vector $W$ is represented as follows:

$$
\begin{gathered}
\boldsymbol{W}(p)=\left(\boldsymbol{V}_{0}(p), \boldsymbol{V}_{1}(p), \ldots, \boldsymbol{V}_{K}(p)\right)^{T}, \\
\boldsymbol{V}_{i}=\left[\rho\left(m=0, \ldots, M ; z_{i}\right), \sigma_{x x}\left(m=0, \ldots, M ; z_{i}\right), \ldots\right. \\
\left.\ldots P\left(m=0, \ldots, M ; z_{i}\right), u_{z}\left(m=0, \ldots, M ; z_{i}\right)\right]^{T} .
\end{gathered}
$$

Then for every $n$-th harmonic $(n=0, \ldots, N)$ the system of linear algebraic equations can be written down in the vector form:

$$
\left(A+\frac{h}{2} E\right) \boldsymbol{W}(p)=\boldsymbol{F}(p-1) .
$$

Note that only the right-hand side of the obtained system of algebraic equations includes the parameter $p$ (the degree of the Laguerre polynomials) and has a recurrent $p$ dependence. The matrix $A$ is thus independent of $p$. A sequence of wave field components in the solution vector $\boldsymbol{V}$ is chosen to minimize the number of diagonals in the matrix $A$. The main diagonal of the matrix has the components of this system multiplied by the parameter $h$ (the Laguerre transform parameter). By changing the parameter $h$, the condition number of the matrix can be considerably improved. Solving the system of linear algebraic equations (20) determines spectral values for all the wave field components $\boldsymbol{W}\left(m, n, z_{i}, p\right)$. Then, using the inversion formulas for the Fourier transform (14), (15), (18), (19), and the Laguerre transform (17), we obtain a solution to the initial problem (8)-(12). In the analytical Fourier and Laguerre transforms, when 
determining functions by their spectra, inversion formulas in the form of infinite sums are used. A necessary condition in the numerical implementation is to determine the number of terms of the summable series to construct a solution with a given accuracy. For instance, the number of harmonics in the inversion formulas of the Fourier transform (14), (15), (18), (19) depends on a minimal spatial wavelength in the medium and on the size of the spatial calculation domain of the field given by the finite limits of the integral transform. In addition, the convergence rate of the summable series depends on smoothness of functions of the wave field. The number of the Laguerre harmonics for determining functions by formula (17) depends on a signal given in the source $f(t)$, the parameter $h$, and the time interval of the wave field. Papers [5-8] consider in detail how one can determine the required number of harmonics and choose an optimal value of the parameter $h$.

\section{Numerical Results}

Figures 1-3 show the results of numerical calculations of a wave field as snapshots at a fixed time for the horizontal component of the displacement velocity $u_{x}(x, y, z)$. Figure 1 presents a snapshot of the wave field for $u_{x}(x, y, z)$ in the plane $X Z$ at the time $t=15 \mathrm{sec}$. This model of the medium consists of a homogeneous elastic layer and an atmospheric layer separated by a plane boundary. The physical characteristics of the layers are as follows:

- the atmosphere: sound speed $c_{p}=340 \mathrm{~m} \cdot \mathrm{sec}^{-1}$. Density versus coordinate $z$ was calculated by the formula $\rho_{0}(z)=\rho_{1} \exp (-z / H) \quad, \quad$ where $\rho_{1}=1.225 \cdot 10^{-3}$ $\mathrm{g} \cdot \mathrm{cm}^{-3}, H=6700 \mathrm{~m}$;

- the elastic layer: pressure wave velocity $c_{p}=300$ $\mathrm{m} \cdot \mathrm{sec}^{-1}$, shear wave velocity $c_{s}=200 \mathrm{~m} \cdot \mathrm{sec}^{-1}$, density $\rho_{0}=1.2 \mathrm{~g} \cdot \mathrm{cm}^{-3}$.

A bounded domain, $(x, y, z)=(15 \mathrm{~km}, 15 \mathrm{~km}, 10 \mathrm{~km})$, was used for the calculations. A wave field from a point source (a pressure center) located in the elastic medium at a depth of $1 / 4$ of the length of a pressure wave with coordinates $\left(x_{0}, y_{0}, z_{0}\right)=(6 \mathrm{~km}, 7.5 \mathrm{~km},-0.075 \mathrm{~km})$ was simulated. The figure shows the wave fields for the horizontal component $u_{x}$ of the displacement velocity in the plane $X Z$ at $y=y_{0}=7.5 \mathrm{~km}$ : without wind (top), with the wind speed in the atmosphere of $50 \mathrm{~m} \cdot \mathrm{sec}^{-1}$ (bottom). The elastic medium-atmosphere interface is shown by the solid line. This figure demonstrates that in the elastic medium, in addition to the spherical P-pressure wave and the conic S-shear wave, there also propagates a "non-ray" spherical wave $\mathrm{S}^{*}$, and then there follows a surface Stoneley-Scholte wave. An acoustic-gravity wave refracted at the Earth-atmosphere boundary propagates in the atmosphere. At the boundary, this wave generates the corresponding pressure and shear waves in
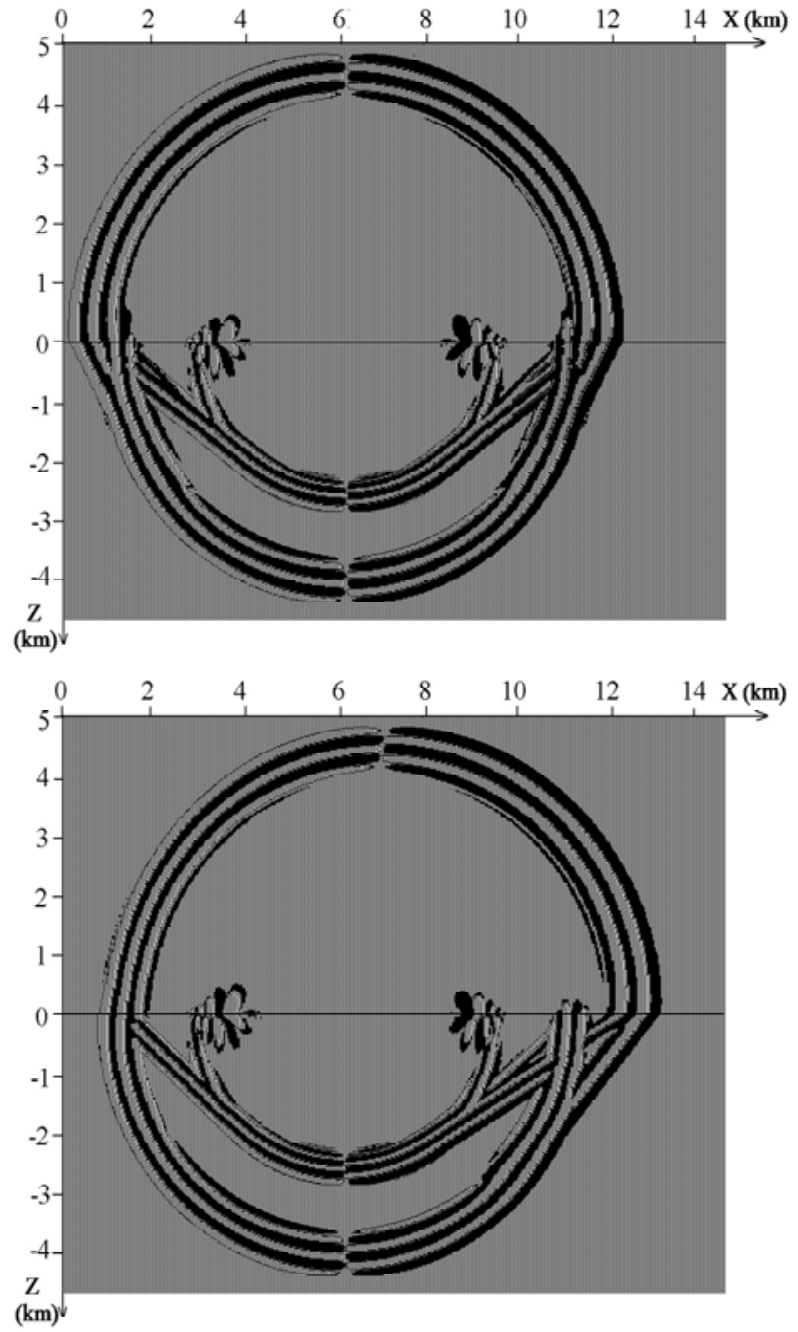

Figure 1. A snapshot at $t=15 \mathrm{sec}$ for the velocity component $u_{x}$ in the plane (XZ) without wind (top), with wind (bottom) (wind speed $50 \mathrm{~m} \cdot \mathrm{sec}^{-1}$ ).

the elastic medium.

Figures 2 and $\mathbf{3}$ present snapshots of the wave field when the seismic waves velocity in the elastic medium is greater than the sound speed in the atmosphere. In this model, the physical characteristics of the elastic medium and the atmosphere are as follows:

- the atmosphere: sound speed $c_{p}=340 \mathrm{~m} \cdot \mathrm{sec}^{-1}$. Density versus coordinate $z$ was calculated by the formula $\rho_{0}(z)=\rho_{1} \exp (-z / H) \quad, \quad$ where $\rho_{1}=1.225 \cdot 10^{-3}$ $\mathrm{g} \cdot \mathrm{cm}^{-3}, H=6700 \mathrm{~m}$;

- the elastic layer: pressure wave velocity $c_{p}=800$ $\mathrm{m} \cdot \mathrm{sec}^{-1}$, shear wave velocity $c_{s}=500 \mathrm{~m} \cdot \mathrm{sec}^{-1}$, density $\rho_{0}=1.5 \mathrm{~g} \cdot \mathrm{cm}^{-3}$.

A bounded domain $(x, y, z)=(20 \mathrm{~km}, 16 \mathrm{~km}, 14 \mathrm{~km})$, was used for the calculations. A wave field from a point source (the pressure center) located in the elastic medium at a depth of $1 / 4$ of the length of a pressure wave with the coordinates $\left(x_{0}, y_{0}, z_{0}\right)=(10 \mathrm{~km}, 8 \mathrm{~km},-0.2 \mathrm{~km})$ was 

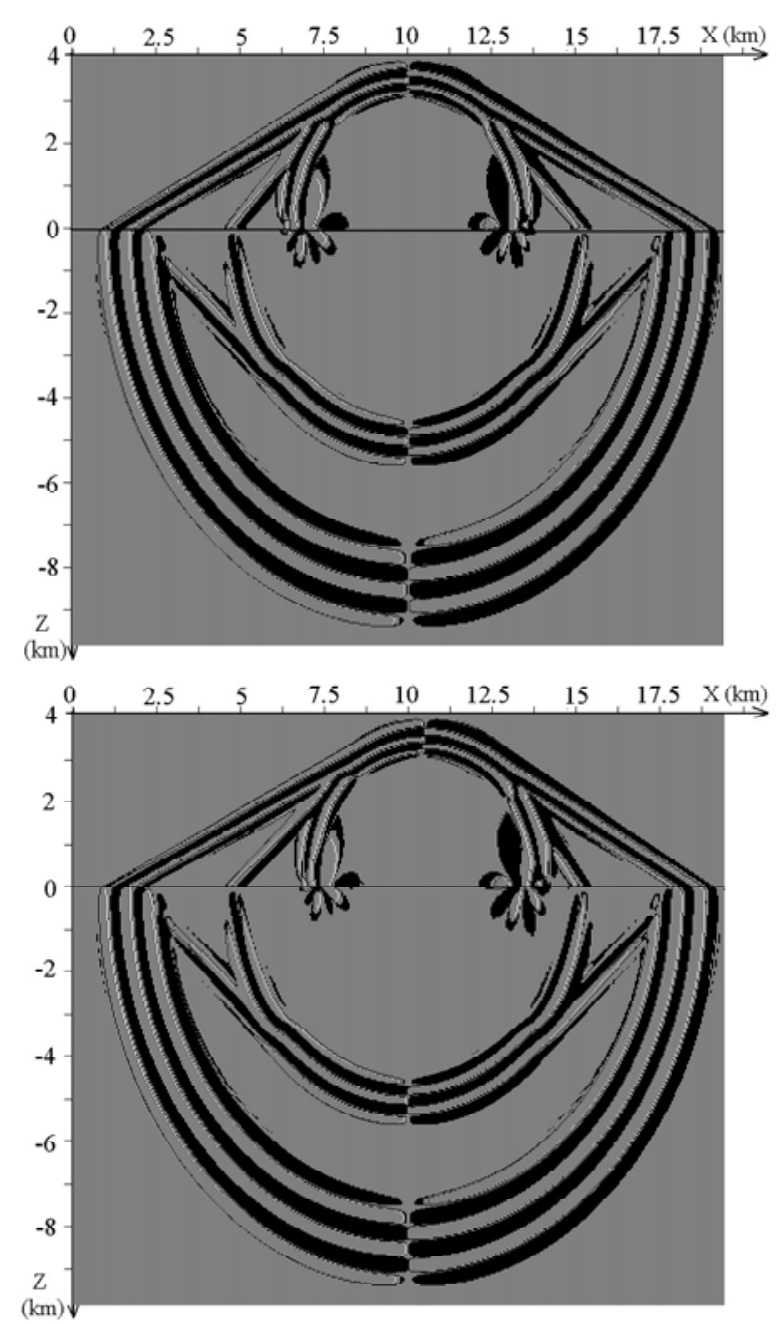

Figure 2. A snapshot at $t=12 \mathrm{sec}$ for the velocity component $\mathrm{u}_{\mathrm{x}}$ in the plane (XZ) without wind (top), with wind (bottom) (wind speed $50 \mathrm{~m} \cdot \mathrm{sec}^{-1}$ ).

simulated.

Figure 2 shows the wave fields for the horizontal component $u_{x}$, of the displacement velocity in the plane $X Z$ at $y=y_{0}=8 \mathrm{~km}$ : without wind (top), with wind speed in the atmosphere of $50 \mathrm{~m} \cdot \mathrm{sec}^{-1}$ (bottom). The elastic medium-atmosphere interface is shown by the solid line. This figure shows that in the atmosphere, in addition to the conical $P$ - pressure wave and the conical $S$-shear wave, there also propagates a "non-ray" spherical wave $P^{*}$, and then there follows a surface StoneleyScholte wave.

Figure 3 presents snapshots of a 3D wave field at $t=10$ sec for the velocity component $u_{x}$ with the wind speed of $50 \mathrm{~m} \cdot \mathrm{sec}^{-1}$ in the atmosphere.

The numerical simulation results have revealed some new peculiarities of the wave propagation with wind in the atmosphere. Specifically, the influence of the wind on the propagation velocity of the surface Stoneley

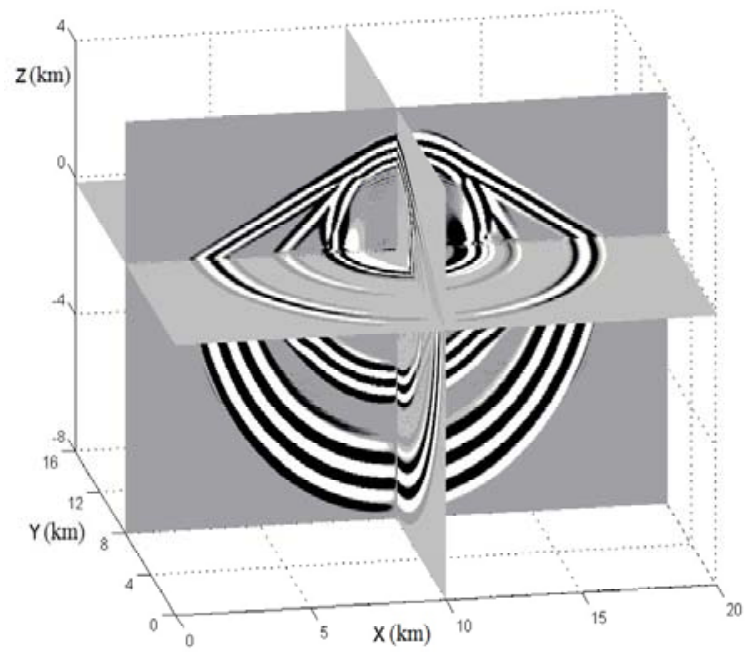

Figure 3. A snapshot of a wave field for the horizontal velocity component $u_{x}(x, y, z)$, at $t=10$ sec with wind in the atmosphere (wind speed $50 \mathrm{~m} \cdot \mathrm{sec}^{-1}$ ).

waves in an elastic medium has been demonstrated. The numerical results have also shown that the velocity of these waves increases downwind and, hence, it decreases upwind by a quantity equal to the wind speed. The same influence of wind is on a non-ray spherical exchange acoustic-gravity wave propagating in the atmosphere from a source located in a solid medium. Another fact of the wind influence that has been established is that the surface wave changes in the amplitude along its front. This manifests itself as an increase in the amplitude in that part of the wave front that propagates downwind and a decrease in the wave front propagating upwind but with conservation of the total wave energy.

\section{Conclusion}

The approach proposed to the statement and solution of the problem makes it possible to simulate the effects of the wave field propagation in a unified mathematical earth-atmosphere model and to study the exchange waves at their boundary. The numerical simulation of these processes makes it possible to investigate the peculiarities of the wind effects on the propagation of the acoustic-gravity atmospheric waves and surface Stoneley waves.

\section{Acknowledgements}

This work was supported by the Russian Foundation for Basic Research (projects no. 11-05-00937, 13-05-00076 and 13-05-12051).

\section{REFERENCES}

[1] A. S. Alekseev, B. M. Glinsky, S. I. Dryakhlov, et al., "The Effect of Acoustic-Seismic Induction in Vibroseis- 
mic Sounding," Dokl. RAN [in Russian], Vol. 346, No. 5, 1996, pp. 664-667.

[2] L. A. Gasilova and Yu. V. Petukhov, "On the Theory of Surface Wave Propagation along Different Interfaces in the Atmosphere," Izv. RAN. Fizika Atmosfery i Okean [in Russian], Vol. 35, No. 1, 1999. pp. 14-23.

[3] A. V. Razin, "Propagation of a Spherical Acoustic Delta Wavelet along the Gas-Solid Interface," Izv. RAN. Fizika zemli [in Russian], No. 2, 1993, pp. 73-77.

[4] B. G. Mikhailenko and G. V. Reshetova, "Mathematical Simulation of Propagation of Seismic and Acoustic-Gravity Waves for an Inhomogeneous Earth-Atmosphere Model," Geologiya i geofizika [in Russian], Vol. 47, No. 5, 2006, pp. 547-556.

[5] B. G. Mikhailenko, "Spectral Laguerre Method for the Approximate Solution of Time Dependent Problems," Applied Mathematics Letters, Vol. 12, No. 4, 1999, pp. 105-110. http://dx.doi.org/10.1016/S0893-9659(99)00043-9

[6] G. V. Konyukh, B. G. Mikhailenko and A. A. Mikhailov, "Application of the Integral Laguerre Transforms for Forward Seismic Modeling," Journal of Computational
Acoustics, Vol. 9, No. 4, 2001, pp. 1523-1541.

[7] B. G. Mikhailenko, A. A. Mikhailov and G. V. Reshetova, "Numerical Modeling of Transient Seismic Fields in Viscoelastic Media Based on the Laguerre Spectral Method," Journal Pure and Applied Geophysics, Vol. 160, No. 7, 2003, pp. 1207-1224. http://dx.doi.org/10.1007/s000240300002

[8] B. G. Mikhailenko, A. A. Mikhailov and G. V. Reshetova, "Numerical Viscoelastic Modeling by the Spectral Laguerre Method," Geophysical Prospecting, Vol. 51, No. 1, 2003, pp. 37-48.

http://dx.doi.org/10.1046/j.1365-2478.2003.00352.x

[9] Kh. Kh. Imomnazarov and A. A. Mikhailov, "Use of the Spectral Laguerre Method to Solve a Linear 2D Dynamic Problem for Porous Media," Sib. Zh. Industr. Matem [in Russian], Vol. 11, No. 2, 2008, pp. 86-95.

[10] P. K. Suetin, "Classical Orthogonal Polynomials," Nauka, Moscow, 1974.

[11] J. Virieux, "P-SV Wave Propagation in Heterogeneous Media: Velocity-Stress Finite-Difference Method," Geophysics, Vol. 51, No. 4, 1986, pp. 889-901. 